
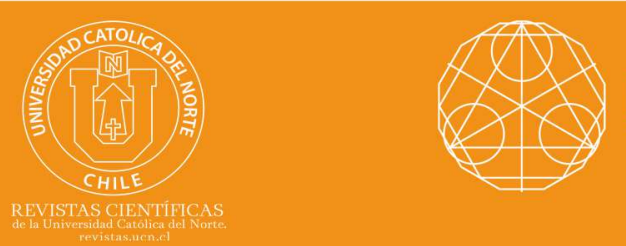

\title{
On even vertex odd mean labeling of the calendula graphs
}

\section{Basher ${ }^{1}$ (1) orcid.org/0000-0003-4576-1586}

${ }^{1}$ Suez University, Dept. of Mathematics and Computer Science, Faculty of Sci., Suez, Egypt. 四 mohamed.beshir@suezuniv.edu.eg

\section{Abstract:}

A graph $G$ with $|E(G)|=q$, an injective function $f: V(G) \rightarrow$ $\{0,2,4, \ldots, 2 q\}$ is an even vertex odd mean labeling of $G$ that induces the values $\frac{f(u)+f(v)}{2}$ for the $q$ pairs of adjacent vertices $u, v$ are distinct. In this paper, we investigate an even vertex labeling for the calendula graphs. Moreover we introduce the definition of arbitrary calendula graph and prove that the arbitrary calendula graphs are also even vertex odd mean graphs.

Keywords: Labeling; Even vertex odd mean labeling; Calendula graph.

$\operatorname{MSC}(2020): 05 C 78$.

\section{Cite this article as (IEEE citation style):}

M. Basher, "On even vertex odd mean labeling of the calendula graphs", Proyecciones (Antofagasta, On line), vol. 39, no. 6, pp. 1515-1535, Dec. 2020, doi: 10.22199/issn.0717-6279-202006-0091.

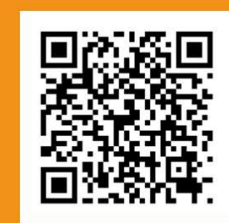

Article copyright: (C) $2020 \mathrm{M}$. Basher. This is an open access article distributed under the terms of the Creative Commons License, which permits unrestricted use and distribution provided the original author and source are credited. 


\section{Introduction}

Unless mentioned or otherwise, the graphs in this paper are finite, undirected and simple. For all other terminology and notations we follow Harary [2].

Let $G$ be a $(p, q)$-graph. Let the vertex set and the edge set of a graph $G$ are denoted by $V(G)$ and $E(G)$ respectively. A graph labeling is an assignment of integers to vertices or edges, or both subject to certain condition. The concept of graph labeling was introduced by Rosa in the late of 1960's [5].The concept of an even vertex odd mean labeling of the graph was introduced by R.Vasuki, A. Nagarajan and S. Arockiaraj [7]. Also they investigated the even vertex odd mean labeling behaviour of some standard graphs. Labeled graphs serve as useful models for a broad rang of applications such as coding theory, mathematical modeling, x-ray, crystallography, radar, Astronomy, circuit design and communication network addressing [1]. We will give brief summery of definitions which are useful for this paper.

Definition 1.1. [7] A function $f$ is called an even vertex odd mean labeling of a graph $G=(V, E)$ if $f: V \rightarrow\{0,2,4, \ldots, 2 q\}$ is injective and the induced function $f^{*}: E \rightarrow\{1,3,5, \ldots, 2 q-1\}$ defined as $f^{*}(e=u v)=$ $\frac{f(u)+f(v)}{2}$ is bijective, $\forall e=u v \in E$.

A graph $G$, which admits an even vertex odd mean labeling is called an even vertex odd mean graph. For more studies see[3,4].

Definition 1.2. [6] Let $m, n \geq 3$. Let $C_{m}$ be a cycle on $m$ vertices. $A$ calendula graph, denoted by $C l_{m, n}$ is a graph constructed from $C_{m}$ and $m$ copies of $C_{n}$ which are $C_{n_{1}}, C_{n_{2}}, \ldots, C_{n_{m}}$ and attaching the i-th edge of $C_{m}$ to an edge of $C_{n_{i}}$ for each $i \in\{1,2,3, \ldots, m\}$. 
Illustration 1.1. In the following Figure 1 we can see $C l_{4,5}$.

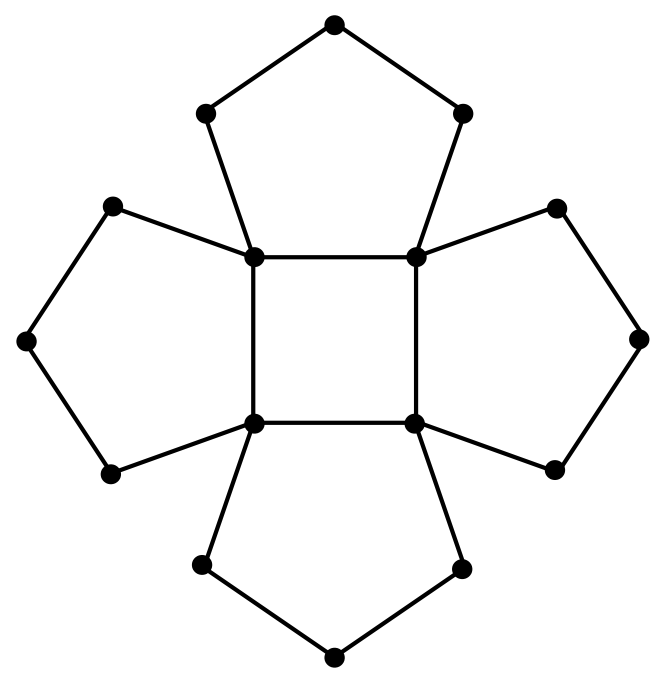

Figure 1.1: A calendula graph $C l_{4,5}$

\section{Calendula graphs and its even vertex odd mean labeling.}

In this section, we show that calendula graph $C l_{m, n}$ for $m$ is an even positive integer and $n \equiv 0(\bmod 4)$ is an even vertex odd mean graph.

Theorem 2.1. Let $m$ and $n$ be two integers with $m$ is an even positive integer and $n \equiv 0(\bmod 4)$. Let $C l_{m, n}$ be a calendula graph, then $C l_{m, n}$ is an even vertex odd mean graph.

Proof. Let $C_{m}$ be a cycle of length $m$, where $m$ is an even positive integer with vertices $u_{1}, u_{2}, \ldots, u_{m}$. Let $C_{n_{i}}, 1 \leq i \leq m$ be $m$ copies of a cycle of length $n$, where $n \equiv 0(\bmod 4)$. Let $v_{i j}, 1 \leq i \leq m, 1 \leq j \leq n$ be the vertices of $m$ copies of $C_{n}$. Let $e_{i}=u_{i} u_{(i+1)}$ denote to the edges of the cycle $C_{m}$ for $1 \leq i \leq m-1$ and $e_{m}=u_{m} u_{1}$. Let $e_{i j}=v_{i j} v_{i(j+1)}$ denote to the edges of $m$ copies of the cycle $C_{n}$ for $1 \leq i \leq m, 1 \leq j \leq n-1$ and $e_{i j}=v_{i n} v_{i 1}$ for $1 \leq i \leq m$. The calendula graphs $C l_{m, n}$ obtained by attaching each edge of $e_{i}$ of $C_{m}$ to an edge $e_{i j}$ of $C_{n_{i}}$ for each $1 \leq i \leq m, 1 \leq j \leq n$. Then it obvious that the order of $C l_{m, n}$ is $m(n-1)$ and the size of $C l_{m, n}$ is $m n$. Define $f: V\left(C l_{m, n}\right) \rightarrow\{0,2,4, \ldots, 2 q-2,2 q=2 m n\}$ as follows:

Case (I): When $m \equiv 0(\bmod 4)$ and $n=4$. 
$\mathrm{f}\left(\mathrm{u}_{i}\right)= \begin{cases}2 n i-2, & 1 \leq i \leq \frac{m}{2} \text { and } i \text { is odd } \\ 2 n i-8, & 2 \leq i \leq \frac{m}{2} \text { and } i \text { is even } \\ 2 n i+2, & \frac{m}{2}+1 \leq i \leq m \text { and } i \text { is odd } \\ 2 n i, & \frac{m}{2}+1 \leq i \leq m \text { and } i \text { is even. }\end{cases}$

For $1 \leq i \leq \frac{m}{2}-1, i$ is odd.

$\mathrm{f}\left(\mathrm{v}_{i j}\right)= \begin{cases}2 n i+2 j-4, & 1 \leq j \leq \frac{n}{2} \text { and } j \text { is odd } \\ 2 n i+2 j-8, & 1 \leq j \leq \frac{n}{2} \text { and } j \text { is even } \\ 2 n i+2 j, & \frac{n}{2}+1 \leq j \leq n \text { and } j \text { is odd } \\ 2 n i+2 j-8, & \frac{n}{2}+1 \leq j \leq n \text { and } j \text { is even. }\end{cases}$

For $1 \leq i \leq \frac{m}{2}-1, i$ is even.

$\mathrm{f}\left(\mathrm{v}_{i j}\right)= \begin{cases}2 n i+2 j-10, & 1 \leq j \leq \frac{n}{2} \text { and } j \text { is odd } \\ 2 n i+2 j-2, & 1 \leq j \leq \frac{n}{2} \text { and } j \text { is even } \\ 2 n i+2 j-6, & \frac{n}{2}+1 \leq j \leq n \text { and } j \text { is odd } \\ 2 n i+2 j-2, & \frac{n}{2}+1 \leq j \leq n \text { and } j \text { is even. }\end{cases}$
$\mathrm{f}\left(\mathrm{v}_{\frac{m}{2}} j\right)= \begin{cases}n m+2 j-10, & 1 \leq j \leq \frac{n}{2} \text { and } j \text { is odd } \\ n m+2, & j=2 \\ n m+2 j+2, & 4 \leq j \leq n \text { and } j \text { is even } \\ n m+2 j-2, & \frac{n}{2}+1 \leq j \leq n \text { and } j \text { is odd. }\end{cases}$

For $\frac{m}{2}+1 \leq i \leq m, i$ is odd.

$\mathrm{f}\left(\mathrm{v}_{i j}\right)= \begin{cases}2 n i+2 j, & 1 \leq j \leq \frac{n}{2} \text { and } j \text { is odd } \\ 2 n i+2 j-4, & 1 \leq j \leq \frac{n}{2} \text { and } j \text { is even } \\ 2 n i+2 j, & \frac{n}{2}+1 \leq j \leq n .\end{cases}$

For $\frac{m}{2}+1 \leq i \leq m, i$ is even.

$\mathrm{f}\left(\mathrm{v}_{i j}\right)= \begin{cases}2 n i+2 j-2, & 1 \leq j \leq \frac{n}{2} \\ 2 n i+2 j-2, & \frac{n}{2}+1 \leq j \leq n \text { and } j \text { is odd } \\ 2 n i+2 j+2, & \frac{n}{2}+1 \leq j \leq n \text { and } j \text { is even. }\end{cases}$

$\mathrm{f}\left(\mathrm{v}_{m j}\right)= \begin{cases}2 n m & j=1 \\ 2 j-6, & 3 \leq j \leq n \text { and } i \text { is odd } \\ 2 j-2, & 2 \leq j \leq n \text { and } i \text { is even. }\end{cases}$

The edge labels of $C l_{m, n}$ are given as follows:

$\mathrm{f}^{*}\left(u_{i} u_{(i+1)}\right)= \begin{cases}2 n i+n-5, & 1 \leq i \leq \frac{m}{2} \\ n m+n-3, & i=\frac{m}{2} \\ 2 n i+n+1, & \frac{m}{2}+1 \leq i \leq m-1 .\end{cases}$

$\mathrm{f}^{*}\left(u_{m} u_{1}\right)=n m+n-1$.

For $1 \leq i \leq \frac{m}{2}-1$.

$\mathrm{f}^{*}\left(v_{i j} v_{i(j+1)}\right)= \begin{cases}2 n i+2 j-5, & 1 \leq j \leq \frac{n}{2} \\ 2 n i+n-3, & j=\frac{n}{2} \\ 2 n i+2 j-3, & \frac{n}{2}+1 \leq j \leq n-1 .\end{cases}$

$\mathrm{f}^{*}\left(v_{i m} u_{i 1}\right)=2 n i+n-5$. 
$\mathrm{f}^{*}\left(v \frac{m}{2} j v \frac{m}{2}(j+1)\right)= \begin{cases}n m-3, & j=1 \\ n m-1, & j=2 \\ n m+2 j-3, & 3 \leq j \leq \frac{n}{2}-1 \\ n m+2 j+1, & \frac{n}{2} \leq j \leq n-1 .\end{cases}$

$\mathrm{f}^{*}\left(v_{\frac{m}{2} n} v \frac{m}{2} 1\right)=n m+n-3$.

For $\frac{m}{2}+1 \leq i \leq m$.

$\mathrm{f}^{*}\left(v_{i j} v_{i(j+1)}\right)= \begin{cases}2 n i+2 j-1, & 1 \leq j \leq \frac{n}{2} \\ 2 n i+2 j+1, & \frac{n}{2}+1 \leq j \leq n-1 .\end{cases}$

$\mathrm{f}^{*}\left(v_{i n} v_{i 1}\right)=2 n i+n+1$.

$\mathrm{f}^{*}\left(v_{m j} v_{m(j+1)}\right)= \begin{cases}n m+1 & j=1 \\ 2 j-3, & 2 \leq j \leq n-1 .\end{cases}$

$\mathrm{f}^{*}\left(v_{m n} v_{m 1}\right)=n m+n-1$.

Case (II): When $m \equiv 0(\bmod 4)$ and $n=4$, we define $f$ as follows:

$$
\mathrm{f}\left(\mathrm{u}_{i}\right)= \begin{cases}8 i-2, & 1 \leq i \leq \frac{m}{2} \text { and } i \text { is odd } \\ 8 i-8, & 2 \leq i \leq \frac{m}{2} \text { and } i \text { is even } \\ 4 m+6, & i=\frac{m}{2}+1 \\ 8 i+2, & \frac{m}{2}+2 \leq i \leq m \text { and } i \text { is odd } \\ 8 i, & \frac{m}{2}+2 \leq i \leq m \text { and } i \text { is even. }\end{cases}
$$

The labels of vertices $v_{i j}$ for $1 \leq i \leq \frac{m}{2}-1,1 \leq j \leq 4$ are given as in the pervious case. Now the labels of remaining vertices $v_{i j}$ for $\frac{m}{2} \leq i \leq m$, $1 \leq j \leq 4$ are given as follows:

$$
\begin{aligned}
& \mathrm{f}\left(\mathrm{v}_{\left(\frac{m}{2}\right) j}\right)= \begin{cases}4 m-8, & j=1 \\
4 m+2, & j=2 \\
4 m+8, & j=3 \\
4 m+6, & j=4 .\end{cases} \\
& \mathrm{f}\left(\mathrm{v}_{\left(\frac{m}{2}+1\right) j}\right)= \begin{cases}4 m+6, & j=1 \\
4 m+12, & j=2 \\
4 m+14, & j=3 \\
4 m+16, & j=4 .\end{cases}
\end{aligned}
$$

For $\frac{m}{2}+2 \leq i \leq m-1, i$ is even.

$$
\mathrm{f}\left(\mathrm{v}_{i j}\right)= \begin{cases}8 i, & j=1 \\ 8 i+2, & j=2 \\ 8 i+4, & j=3 \\ 8 i+10, & j=4 .\end{cases}
$$

For $\frac{m}{2}+2 \leq i \leq m-1, i$ is odd. 


$$
\begin{array}{r}
\mathrm{f}\left(\mathrm{v}_{i j}\right)= \begin{cases}8 i+2, & j=1 \\
8 i, & j=2 \\
8 i+6, & j=3 \\
8 i+8, & j=4 .\end{cases} \\
\mathrm{f}\left(\mathrm{v}_{m j}\right)= \begin{cases}2 n m, & j=1 \\
2, & j=2 \\
0, & j=3 \\
6, & j=4 .\end{cases}
\end{array}
$$

Then, the edge labels of $C l_{m, 4}$ are given as follows:

$\mathrm{f}^{*}\left(u_{i} u_{(i+1)}\right)= \begin{cases}8 i-5, & 1 \leq i \leq \frac{m}{2}-1 \\ 4 m-1, & i=\frac{m}{2} \\ 4 m+11, & i=\frac{m}{2}+1 \\ 8 i+5, & \frac{m}{2}+2 \leq i \leq m-1 .\end{cases}$

$\mathrm{f}^{*}\left(u_{m} u_{1}\right)=4 m+3$.

The labels of edges $v_{i j} v_{i(j+1)}$ for $1 \leq i \leq \frac{m}{2}-1,1 \leq j \leq 3$ and $v_{i 3} v_{i 1}$ for $1 \leq i \leq \frac{m}{2}-1$ are given as in pervious case. Otherwise, the labels of remaining edges $v_{i j} v_{i(j+1)}$ for $\frac{m}{2} \leq i \leq m, 1 \leq j \leq 3$ and $v_{i 4} v_{i 1}$ for $\frac{m}{2} \leq i \leq m$ are given as follows:

$$
\mathrm{f}^{*}\left(v_{\left(\frac{m}{2}\right) j} v_{\left(\frac{m}{2}\right)(j+1)}\right)= \begin{cases}4 m-3, & j=1 \\ 4 m+5, & j=2 \\ 4 m+7, & j=3 .\end{cases}
$$

$\mathrm{f}^{*}\left(v_{\left(\frac{m}{2}\right) 4} v_{\left(\frac{m}{2}\right) 1}\right)=4 m-1$.

$$
\mathrm{f}^{*}\left(v_{\left(\frac{m}{2}+1\right) j} v_{\left(\frac{m}{2}+1\right)(j+1)}\right)= \begin{cases}4 m+9, & j=1 \\ 4 m+13, & j=2 \\ 4 m+15, & j=3 .\end{cases}
$$

$\mathrm{f}^{*}\left(v_{\left(\frac{m}{2}+1\right) 4} v_{\left(\frac{m}{2}+1\right) 1}\right)=4 m+11$.

For $\frac{m}{2}+2 \leq i \leq m-1$.

$\mathrm{f}^{*}\left(v_{i j} v_{i(j+1)}\right)= \begin{cases}8 i+1, & j=1 \\ 8 i+3, & j=2 \\ 8 i+7, & j=3 .\end{cases}$

$\mathrm{f}^{*}\left(v_{i 4} v_{i 1}\right)=8 i+5$.

$\mathrm{f}^{*}\left(v_{m j} v_{m(j+1)}\right)= \begin{cases}4 m+1, & j=1 \\ 1, & j=2 \\ 3, & j=3 .\end{cases}$

$\mathrm{f}^{*}\left(v_{m 4} v_{m 1}\right)=4 m+3$. 
Case (III): When $m=2 k$ and $k$ is odd, we define $f$ as follows:

$$
\mathrm{f}\left(\mathrm{u}_{i}\right)=\left\{\begin{array}{l}
2 n i-2, \quad 1 \leq i \leq \frac{m}{2}-1 \text { and } i \text { is odd } \\
2 n i-8, \quad 2 \leq i \leq \frac{m}{2}-1 \text { and } i \text { is even } \\
2 n i+2, \quad \frac{m}{2} \leq i \leq m \text { and } i \text { is odd } \\
2 n i, \quad \frac{m}{2} \leq i \leq m \text { and } i \text { is even. }
\end{array}\right.
$$

The labels of vertices $v_{i j}$ for $1 \leq i \leq \frac{m}{2}-2,1 \leq j \leq n$ and $v_{m j}$ for $1 \leq j \leq n$ are given as in Case(I). Now the labels of remaining vertices $v_{i j}$ for $\frac{m}{2}-1 \leq i \leq m-1,1 \leq j \leq n$ are given as follows:

$$
\begin{aligned}
& \mathrm{f}\left(\mathrm{v}_{\left(\frac{m}{2}-1\right) j}\right)= \begin{cases}n m-2 n+2 j-10, & 1 \leq j \leq n \text { and } j \text { is odd } \\
n m-2 n+2 j-2, & 1 \leq j \leq \frac{n}{2} \text { and } j \text { is even } \\
n m-2 n+2 j+2, & \frac{n}{2}+2 \leq j \leq n \text { and } j \text { is even. }\end{cases} \\
& \mathrm{f}\left(\mathrm{v}_{\frac{m}{2}} j\right)= \begin{cases}n m+2 j, & 1 \leq j \leq \frac{n}{2} \text { and } j \text { is odd } \\
n m-8, & \mathrm{j}=2 \\
n m+2 j-8, & 4 \leq j \leq \frac{n}{2} \text { and } j \text { is even } \\
n m+2 j, & \frac{n}{2}+1 \leq j \leq n .\end{cases}
\end{aligned}
$$

For $\frac{m}{2}+1 \leq i \leq m-1, i$ is even.

$$
\mathrm{f}\left(\mathrm{v}_{i j}\right)= \begin{cases}2 n i+2 j-2, & 1 \leq j \leq \frac{n}{2} \\ 2 n i+2 j-2, & \frac{n}{2}+1 \leq j \leq n \text { and } j \text { is odd } \\ 2 n i+2 j+2, & \frac{n}{2}+1 \leq j \leq n \text { and } j \text { is even. }\end{cases}
$$

For $\frac{m}{2}+1 \leq i \leq m-1, i$ is odd.

$$
\mathrm{f}\left(\mathrm{v}_{i j}\right)= \begin{cases}2 n i+2 j, & 1 \leq j \leq \frac{n}{2} \text { and } j \text { is odd } \\ 2 n i+2 j-4, & 1 \leq j \leq \frac{n}{2} \text { and } j \text { is even } \\ 2 n i+2 j, & \frac{n}{2}+1 \leq j \leq n .\end{cases}
$$

Hence, the edge labels of $C l_{m n}$ are given as follows:

$$
\begin{aligned}
& \mathrm{f}^{*}\left(u_{i} u_{(i+1)}\right)= \begin{cases}2 n i-5, & 1 \leq i \leq \frac{m}{2}-2 \\
2 n i-3, & i=\frac{m}{2}-1 \\
2 n i+1, & \frac{m}{2} \leq i \leq m-1 .\end{cases} \\
& \mathrm{f}^{*}\left(u_{m} u_{1}\right)=n m-n-1 .
\end{aligned}
$$

The labels of edges $v_{i j} v_{i(j+1)}$ for $1 \leq i \leq \frac{m}{2}-2,1 \leq j \leq n-1$, $v_{i n} v_{i 1}$ for $1 \leq i \leq \frac{m}{2}-2, v_{m j} v_{m(j+1)}$ for $1 \leq j \leq n-1$ and $v_{m n} v_{m 1}$ are given as in Case(I). Otherwise, the labels of remaining edges $v_{i j} v_{i(j+1)}$ for $\frac{m}{2}-1 \leq i \leq m-1,1 \leq j \leq n-1$ and $v_{i n} v_{i 1}$ for $\frac{m}{2}-1 \leq i \leq m-1$ are given as follows:

$$
\begin{aligned}
& \mathrm{f}^{*}\left(v_{\left(\frac{m}{2}-1\right) j} v_{\left(\frac{m}{2}-1\right)(j+1)}\right)= \begin{cases}n m-2 n+2 j-5, & 1 \leq j \leq \frac{n}{2} \\
n m-2 n+2 j-3, & \frac{n}{2}+1 \leq j \leq n-1 .\end{cases} \\
& \mathrm{f}^{*}\left(v_{\left(\frac{m}{2}-1\right) n} v_{\left(\frac{m}{2}-1\right) 1}\right)=n m-n-3 .
\end{aligned}
$$




$$
\mathrm{f}^{*}\left(v \frac{m}{2} j v \frac{m}{2}(j+1)\right)= \begin{cases}n m-3, & j=1 \\ n m-5, & j=2 \\ n m+2 j-3, & 3 \leq j \leq \frac{n}{2} \\ n m+2 j+1, & \frac{n}{2}+1 \leq j \leq n-1 .\end{cases}
$$

$\mathrm{f}^{*}\left(v_{\frac{m}{2} n} n v_{\frac{m}{2}} 1\right)=n m+n+1$.

For $\frac{m}{2}+1 \leq i \leq m-1$.

$$
\begin{aligned}
& \mathrm{f}^{*}\left(v_{i j} v_{i(j+1)}\right)= \begin{cases}2 n i+2 j-1, & 1 \leq j \leq \frac{n}{2} \\
2 n i+n-1, & j=\frac{n}{2} \\
2 n i+2 j+1, & \frac{n}{2}+1 \leq j \leq n-1 .\end{cases} \\
& \mathrm{f}^{*}\left(v_{i n} v_{i 1}\right)=2 n i+n+1 .
\end{aligned}
$$

Illustration 2.1. In the following Figure 2, Figure 3 and Figure 4 we show an even vertex odd mean labeling on calendula graphs $C l_{8,8}, C l_{8,4}$ and $C l_{6,8}$ respectively.

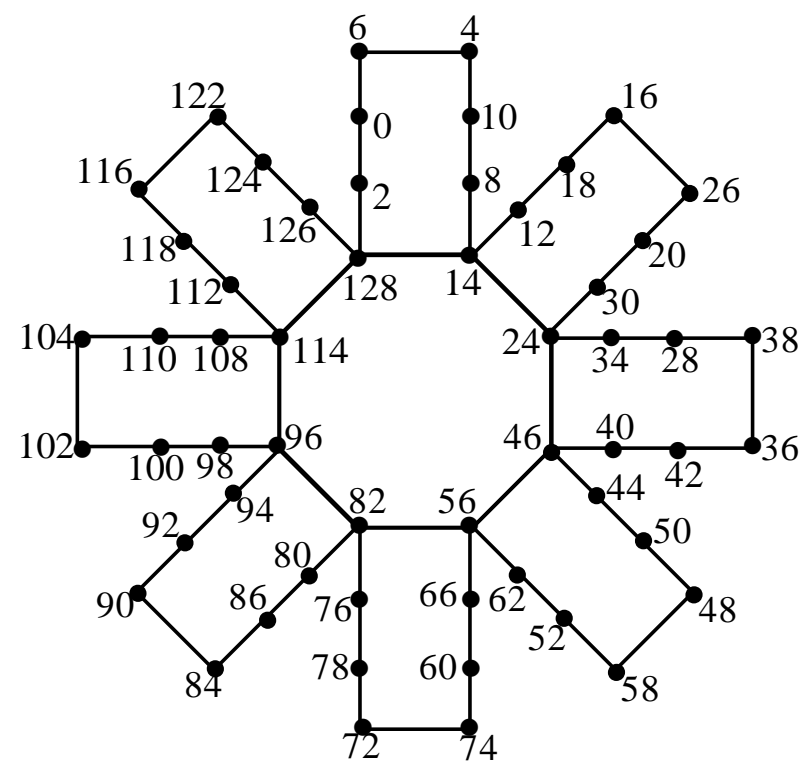

Figure 2.1: An even vertex odd mean graph of $C l_{8,8}$ 


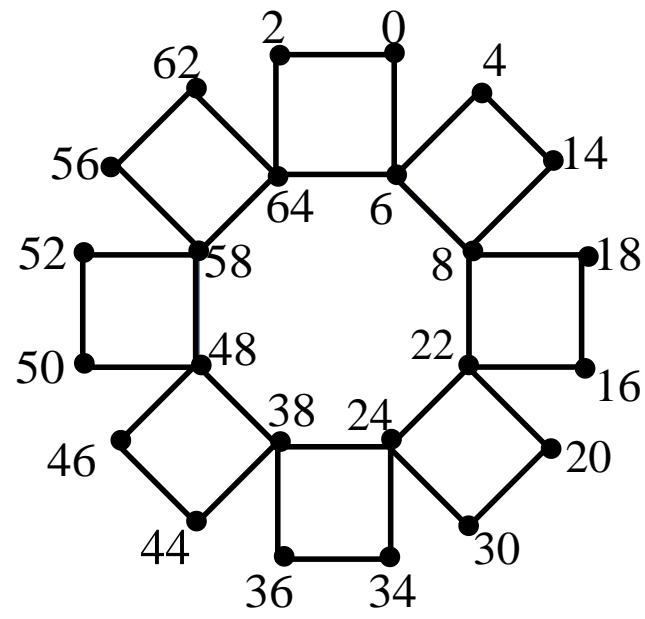

Figure 2.2: An even vertex odd mean graph of $C l_{8,4}$

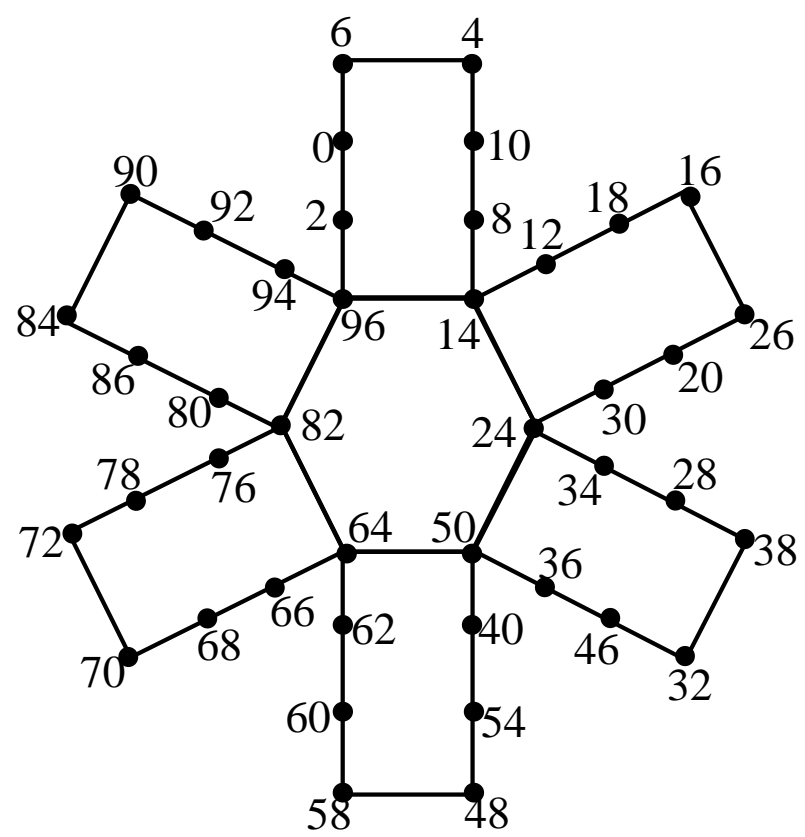

Figure 2.3: An even vertex odd mean graph of $C l_{6,8}$ 


\section{Arbitrary calendula graphs and its even vertex odd mean labeling.}

In this section, we introduce the definition of arbitrary calendula graph and prove that the arbitrary calendula graph is an even vertex odd mean graph.

Definition 3.1. A calendula graph is said to be arbitrary calendula graph, denoted by $A C l_{\left(m ; n_{1}, n_{2}, \ldots, n_{m}\right)}$ if every edge from $C_{m}$ is attached by an edge from arbitrary $C_{n_{i}}$ where $n_{i}$ may vary for each $1 \leq i \leq m$.

Illustration 3.1. In the following Figure 5 the arbitrary calendula graph $A C l_{(6 ; 6,5,4,3,4,5)}$ is shown where $m=6, n_{1}=6, n_{2}=5, n_{3}=4, n_{4}=3, n_{5}=$ 4 and $n_{6}=5$.

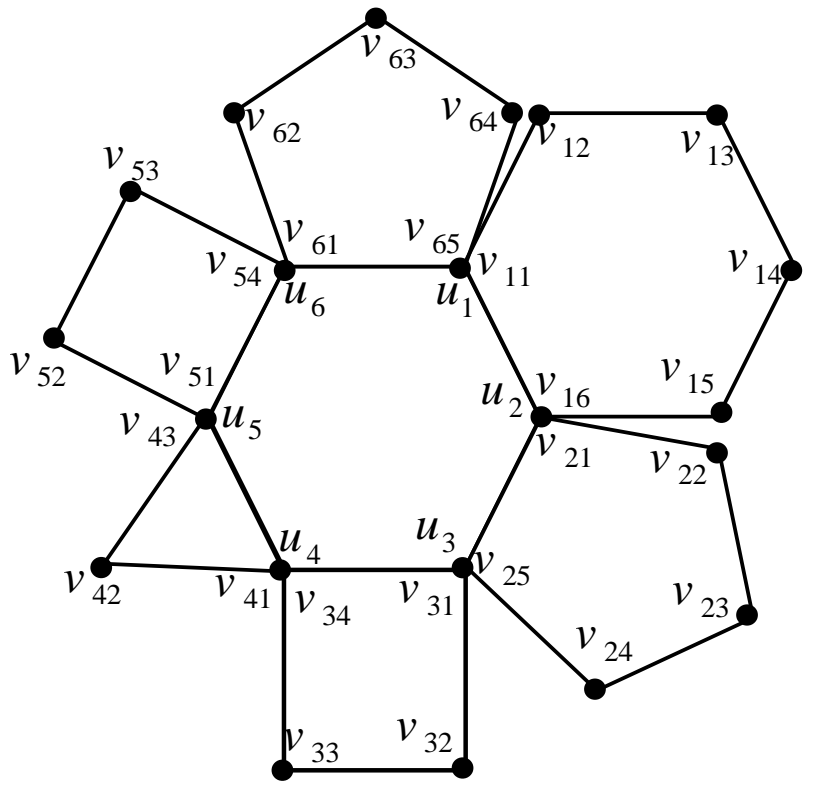

Figure 3.1: Arbitrary calendula graph $A C l_{(6 ; 6,5,4,3,4,5)}$ 
Theorem 3.2. Let $m$ and $n_{i}, 1 \leq i \leq m$ be $m+1$ integers with $m$ is an even positive integer and $n_{i} \equiv 0(\bmod 4), 1 \leq i \leq m$ such that $n_{\frac{m}{2}}=n_{m}$ and $\sum_{i=1}^{\frac{m}{2}} n_{i}=\sum_{i=\frac{m}{2}+1}^{m} n_{i}$, then the arbitrary calendula graph $A C l_{\left(m ; n_{1}, n_{2}, \ldots, n_{m}\right)}$ is an even vertex odd mean graph.

Proof. Let $C_{m}: u_{1} u_{2} \ldots u_{m} u_{1}$ be the cycle of length $m$, where $m$ is an even positive integer. Let $C_{n_{i}}, 1 \leq i \leq m$ be $m$ arbitrary cycles such that $n_{\frac{m}{2}}=n_{m}$ and $\sum_{i=1}^{\frac{m}{2}} n_{i}=\sum_{i=\frac{m}{2}+1}^{m} n_{i}$, where $n_{i}$ may vary for each $1 \leq i \leq m$ and $n_{i} \equiv 0(\bmod 4)$. Let $e_{i}=u_{i} u_{(i+1)}$ denote to the edges of the cycle $C_{m}$ for $1 \leq i \leq m-1$ and $e_{m}=u_{m} u_{1}$. Let $e_{i j}=v_{i j} v_{i(j+1)}$ denote to the edges of $m$ arbitrary cycles $C_{n_{i}}$ for $1 \leq i \leq m, 1 \leq j \leq n_{i}-1$ and $e_{i j}=v_{i n_{i}} v_{i 1}$ for $1 \leq i \leq m$. The arbitrary calendula graphs $A C l_{\left(m ; n_{1}, n_{2}, \ldots, n_{m}\right)}$ obtained by attaching each edge of $e_{i}$ of $C_{m}$ to an edge $e_{i j}$ of $C_{n_{i}}$ for each $1 \leq i \leq m, 1 \leq j \leq n$. Then it is clear that the number of vertices of the graph $A C l_{\left(m ; n_{1}, n_{2}, \ldots, n_{m}\right)}$ is $\sum_{i=1}^{m} n_{i}-m$ and the number of edges of the graph $A C l_{\left(m ; n_{1}, n_{2}, \ldots, n_{m}\right)}$ is $\sum_{i=1}^{m} n_{i}$. We define $f: V\left(A C l_{\left(m ; n_{1}, n_{2}, \ldots, n_{m}\right)}\right) \rightarrow$ $\left\{0,2,4, \ldots, 2 q-2,2 q=2 \sum_{i=1}^{m} n_{i}\right\}$ as follows:

Case (I): When $m \equiv 0(\bmod 4)$ and $n_{m}=n_{\frac{m}{2}}=4$.

$$
\mathrm{f}\left(\mathrm{u}_{i}\right)= \begin{cases}2 n_{m}-2, & i=1 \\ 2 n_{m}+2 \sum_{k=1}^{i-1} n_{k}-2, & 2 \leq i \leq \frac{m}{2} \text { and } i \text { is odd } \\ 2 n_{m}+2 \sum_{k=1}^{i-1} n_{k}-8, & 2 \leq i \leq \frac{m}{2} \text { and } i \text { is even } \\ 2 n_{m}+2 \sum_{k=1}^{i-1} n_{k}+2, & \frac{m}{2}+1 \leq i \leq m-1 \text { and } i \text { is odd } \\ 2 n_{m}+2 \sum_{k=1}^{i-1} n_{k}-4, & \frac{m}{2}+1 \leq i \leq m-1 \text { and } i \text { is even } \\ 2 \sum_{k=1}^{m} n_{k}, & i=m \\ 2 n_{m}+2 j-4, & 1 \leq j \leq \frac{n_{1}}{2} \text { and } j \text { is odd } \\ 2 n_{m}+2 j-8, & 1 \leq j \leq n_{1} \text { and } j \text { is even } \\ 2 n_{m}+2 j, & \frac{n_{1}}{2}+1 \leq j \leq n_{1} \text { and } j \text { is odd. }\end{cases}
$$

For $2 \leq i \leq \frac{m}{2}-1, i$ is odd. 


$$
\mathrm{f}\left(\mathrm{v}_{i j}\right)=\left\{\begin{array}{lll}
2 n_{m}+2 \sum_{k=1}^{i-1} n_{k}+2 j-4, & 2 \leq j \leq \frac{n_{i}}{2} \text { and } j \text { is odd } \\
2 n_{m}+2 \sum_{k=1}^{i-1} n_{k}+2 j-8, & 2 \leq j \leq \frac{n_{i}}{2} \text { and } j \text { is even } \\
2 n_{m}+2 \sum_{k=1}^{i-1} n_{k}+2 j, & \frac{n_{i}}{2}+1 \leq j \leq n_{i} \text { and } j \text { is odd } \\
2 n_{m}+2 \sum_{k=1}^{i-1} n_{k}+2 j-8, & \frac{n_{i}}{2}+1 \leq j \leq n_{i} \text { and } j \text { is even. }
\end{array}\right.
$$

For $2 \leq i \leq \frac{m}{2}-1, i$ is even.

$$
\mathrm{f}\left(\mathrm{v}_{i j}\right)=\left\{\begin{array}{lc}
2 n_{m}+2 \sum_{k=1}^{i-1} n_{k}+2 j-10, & 2 \leq j \leq \frac{n_{i}}{2} \text { and } j \text { is odd } \\
2 n_{m}+2 \sum_{k=1}^{i-1} n_{k}+2 j-2, & 2 \leq j \leq \frac{n_{i}}{2} \text { and } j \text { is even } \\
2 n_{m}-2 n_{i}+2 \sum_{k=1}^{i} n_{k}+2 j-6, & \frac{n_{i}}{2}+1 \leq j \leq n_{i} \text { and } j \text { is odd } \\
2 n_{m}-2 n_{i}+2 \sum_{k=1}^{i} n_{k}+2 j-2, & \frac{n_{i}}{2}+1 \leq j \leq n_{i} \text { and } j \text { is even. }
\end{array}\right.
$$

For $\frac{m}{2}+1 \leq i \leq m-2, i$ is odd.

$$
\mathrm{f}\left(\mathrm{v}_{i j}\right)= \begin{cases}2 n_{m}+2 \sum_{k=1}^{i-1} n_{k}+2 j, & 1 \leq j \leq \frac{n_{i}}{2} \text { and } j \text { is odd } \\ 2 n_{m}+2 \sum_{k=1}^{i-1} n_{k}+2 j-4, & 1 \leq j \leq \frac{n_{i}}{2} \text { and } j \text { is even } \\ 2 n_{m}-2 n_{i}+\sum_{k=1}^{i} n_{k}+2 j+4, & \frac{n_{i}}{2}+1 \leq j \leq n_{i} \text { and } j \text { is odd } \\ 2 n_{m}-2 n_{i}+\sum_{k=1}^{i} n_{k}+2 j-4, & \frac{n_{i}}{2}+1 \leq j \leq n_{i} \text { and } j \text { is even. }\end{cases}
$$

For $\frac{m}{2}+1 \leq i \leq m-2, i$ is even. 


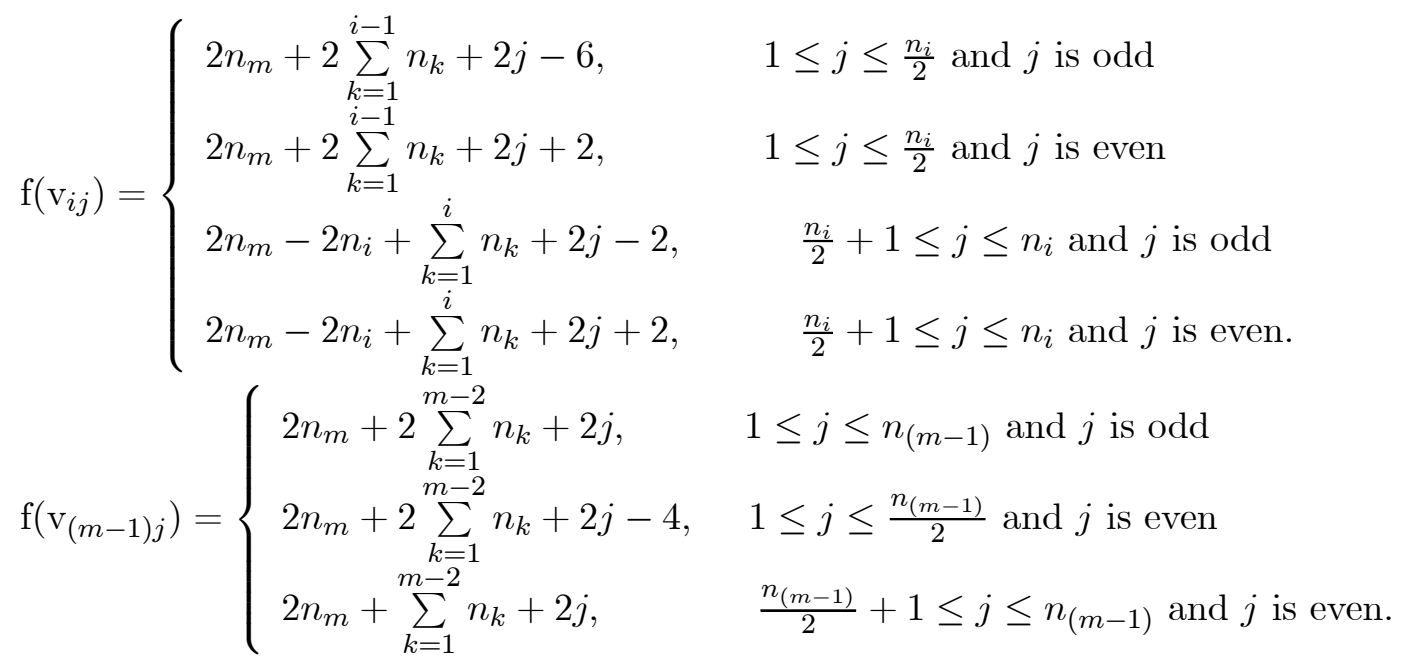

$\mathrm{f}\left(\mathrm{v}_{m j}\right)=\left\{\begin{array}{cc}2 \sum_{k=1}^{m} n_{k} & j=1 \\ 2 j-6, & 3 \leq j \leq n_{m} \text { and } j \text { is odd } \\ 2 j-2, & 2 \leq j \leq n_{m} \text { and } j \text { is even. }\end{array}\right.$

Thus, the edge labels of $A C l_{\left(m ; n_{1}, n_{2}, \ldots, n_{m}\right)}$ are given as follows:

$f^{*}\left(u_{i} u_{(i+1)}\right)= \begin{cases}2 n_{m}+n_{1}-5, & i=1 \\ 2 n_{m}+2 \sum_{k=1}^{i-1} n_{k}+n_{i}-5, & 2 \leq i \leq \frac{m}{2}-1 \\ 2 n_{m}+2 \sum_{k=1}^{\frac{m}{2}-1} n_{k}+n_{\frac{m}{2}}-3, & i=\frac{m}{2} \\ 2 n_{m}+2 \sum_{k=1}^{i-1} n_{k}+n_{i}-1, & \frac{m}{2}+1 \leq i \leq m-1 .\end{cases}$

$\mathrm{f}^{*}\left(u_{m} u_{1}\right)=n_{m}+2 \sum_{k=1}^{m} n_{k}-1$.

$\mathrm{f}^{*}\left(v_{1 j} v_{1(j+1)}\right)= \begin{cases}2 n_{m}+2 j-5, & 1 \leq j \leq \frac{n_{1}}{2} \\ 2 n_{m}+2 j-3, & \frac{n_{1}}{2}+1 \leq j \leq n_{1}-1 .\end{cases}$

$\mathrm{f}^{*}\left(v_{1 n_{1}} v_{11}\right)=2 n_{m}+n_{1}-5$.

For $2 \leq i \leq \frac{m}{2}-1$.

$\mathrm{f}^{*}\left(v_{i j} v_{i(j+1)}\right)= \begin{cases}2 n_{m}+2 \sum_{k=1}^{i-1} n_{k}+2 j-5, & 1 \leq j \leq \frac{n_{i}}{2}-1 \\ 2 n_{m}+2 \sum_{k=1}^{i-1} n_{k}+n_{i}-3, & j=\frac{n_{i}}{2} \\ 2 n_{m}+2 \sum_{k=1}^{i-1} n_{k}+2 j-3, & \frac{n_{i}}{2}+1 \leq j \leq n_{i}-1 .\end{cases}$

$\mathrm{f}^{*}\left(v_{i n_{i}} v_{i 1}\right)=2 n_{m}+2 \sum_{k=1}^{i-1} n_{k}+n_{i}-5$. 


$$
\mathrm{f}^{*}\left(v_{\frac{m}{2}} j v_{\frac{m}{2}}(j+1)\right)= \begin{cases}2 n_{m}+2 \sum_{k=1}^{\frac{m}{2}-1} n_{k}-3, & j=1 \\ 2 n_{m}+2 \sum_{k=1}^{\frac{m}{2}-1} n_{k}-1, & j=2 \\ 2 n_{m}+2 \sum_{k=1}^{\frac{m}{2}-1} n_{k}+2 j-3, & 3 \leq j \leq \frac{n \frac{m}{2}}{2}-1 \\ 2 n_{m}+2 \sum_{k=1}^{\frac{m}{2}-1} n_{k}+2 j+1, & \frac{n \frac{m}{2}}{2} \leq j \leq n_{\frac{m}{2}}-1 . \\ \mathrm{f}^{*}\left(v_{\frac{m}{2} n \frac{m}{2}} v_{\frac{m}{2}}\right)=2 n_{m}+2 \sum_{k=1}^{\frac{m}{2}-1} n_{k}+n_{\frac{m}{2}}-3 . & \end{cases}
$$

For $\frac{m}{2}+1 \leq i \leq m-2$.

$$
\begin{aligned}
& \mathrm{f}^{*}\left(v_{i j} v_{i(j+1)}\right)= \begin{cases}2 n_{m}+2 \sum_{k=1}^{i-1} n_{k}+2 j-1, & 1 \leq j \leq \frac{n_{i}}{2}-1 \\
2 n_{m}+2 \sum_{k=1}^{i-1} n_{k}+2 j+1, & \frac{n_{i}}{2} \leq j \leq n_{i}-1 .\end{cases} \\
& \mathrm{f}^{*}\left(v_{i n_{i}} v_{i 1}\right)=2 n_{m}-2 n_{i}+\sum_{k=1}^{i-1} n_{k}+n_{i}-1 .
\end{aligned}
$$$$
\mathrm{f}^{*}\left(v_{(m-1) j} v_{(m-1)(j+1)}\right)= \begin{cases}2 n_{m}+2 \sum_{k=1}^{m-2} n_{k}+2 j-1, & 1 \leq j \leq \frac{n_{(m-1)}}{2}, \\ 2 n_{m}+2 \sum_{k=1}^{m-2} n_{k}+2 j+1, & \frac{n_{(m-1)}}{2}+1 \leq j \leq n_{(m-1)}-1 .\end{cases}
$$$$
\mathrm{f}^{*}\left(v_{(m-1) n_{(m-1)}} v_{(m-1) 1}\right)=2 n_{m}+2 \sum_{k=1}^{m-2} n_{k}+n_{(m-1)}+1 \text {. }
$$$$
\mathrm{f}^{*}\left(v_{m j} v_{m(j+1)}\right)= \begin{cases}\sum_{k=1}^{m} n_{k}+1, & j=1 \\ 2 j-3, & 2 \leq j \leq n_{m}-1 .\end{cases}
$$$$
\mathrm{f}^{*}\left(v_{m n_{m}} v_{m 1}\right)=\sum_{k=1}^{m} n_{k}+n_{m}-1 \text {. }
$$

Case (II): When $m \equiv 0(\bmod 4)$ and $n_{m}=n_{\frac{m}{2}}=4$, we define $f$ as follows:

$$
\mathrm{f}\left(\mathrm{u}_{i}\right)= \begin{cases}6, & i=1 \\ 2 \sum_{k=1}^{i-1} n_{k}+6, & 2 \leq i \leq m-1 \text { and } i \text { is odd } \\ 2 \sum_{k=1}^{i-1} n_{k}, & 2 \leq i \leq \frac{m}{2}+1 \text { and } i \text { is even } \\ 2 \sum_{k=1}^{i-1} n_{k}+8, & \frac{m}{2}+2 \leq i \leq m-1 \text { and } i \text { is even } \\ 2 \sum_{k=1}^{m} n_{k}, & i=m .\end{cases}
$$


The labels of vertices $v_{i j}$ for $1 \leq i \leq \frac{m}{2}-1,1 \leq j \leq n_{i}$ are given as in the pervious case. Now the labels of remaining vertices $v_{i j}$ for $\frac{m}{2} \leq i \leq m$, $1 \leq j \leq n_{i}$ are given as follows:

$$
\mathrm{f}\left(\mathrm{v} \frac{m}{2} j\right)= \begin{cases}2 \sum_{k=1}^{\frac{m}{2}-1} n_{k}, & \mathrm{j}=1 \\ 2 \sum_{k=1}^{\frac{m}{2}-1} n_{k}+10, & \mathrm{j}=2 \\ 2 \sum_{k=1}^{\frac{m}{2}-1} n_{k}+16, & \mathrm{j}=3 \\ 2 \sum_{k=1}^{\frac{m}{2}-1} n_{k}+14, & \mathrm{j}=4 . \\ \mathrm{f}\left(\mathrm{v}\left(\frac{m}{2}+1\right) j\right)= \begin{cases}2 \sum_{k=1}^{\frac{m}{2}} n_{k}+2 j+4, & 1 \leq j \leq \frac{n_{\left(\frac{m}{2}+1\right)}}{2} \text { and } j \text { is odd } \\ 2 \sum_{k=1}^{\frac{m}{2}} n_{k}+2 j+8, & 1 \leq j \leq \frac{n_{\left(\frac{m}{2}+1\right)}}{2} \text { and } j \text { is even } \\ 2 \sum_{k=1}^{\frac{m}{2}} n_{k}+2 j+8, & \frac{n_{\left(\frac{m}{2}+1\right)}}{2}+1 \leq j \leq n_{\left(\frac{m}{2}+1\right)} .\end{cases} \end{cases}
$$

For $\frac{m}{2}+2 \leq i \leq m-1, i$ is even.

$$
\mathrm{f}\left(\mathrm{v}_{i j}\right)=\left\{\begin{array}{cl}
2 \sum_{k=1}^{i-1} n_{k}+2 j+6, & 1 \leq j \leq \frac{n_{i}}{2} \\
2 \sum_{\substack{i-1 \\
i=1}}^{i-1} n_{k}+2 j+10, & \frac{n_{i}}{2}+1 \leq j \leq n_{i} \text { and } j \text { is odd } \\
2 \sum_{k=1}^{i-1} n_{k}+2 j+6, & \frac{n_{i}}{2}+1 \leq j \leq n_{i} \text { and } j \text { is even. }
\end{array}\right.
$$

For $\frac{m}{2}+2 \leq i \leq m-1, i$ is odd.

$$
\begin{aligned}
& \mathrm{f}\left(\mathrm{v}_{i j}\right)= \begin{cases}2 \sum_{k=1}^{i-1} n_{k}+2 j+4, & 1 \leq j \leq \frac{n_{i}}{2} \text { and } j \text { is odd } \\
2 \sum_{k=1}^{i-1} n_{k}+2 j+8, & 1 \leq j \leq \frac{n_{i}}{2} \text { and } j \text { is even } \\
2 \sum_{k=1}^{i} n_{k}-2 n_{i}+2 j+8, & \frac{n_{i}}{2}+1 \leq j \leq n_{i} .\end{cases} \\
& \mathrm{f}\left(\mathrm{v}_{m j}\right)= \begin{cases}2 \sum_{k=1}^{m} n_{k}, & j=1 \\
2, & j=2 \\
0, & j=3 \\
6, & j=4 .\end{cases}
\end{aligned}
$$

Thus, the edge labels of $A C l_{\left(m ; n_{1}, n_{2}, \ldots, n_{m}\right)}$ are given as follows: 


$$
\begin{aligned}
& \mathrm{f}^{*}\left(u_{i} u_{(i+1)}\right)= \begin{cases}n_{1}+3, & i=1 \\
2 \sum_{k=1}^{i-1} n_{k}+n_{i}+3, & 2 \leq i \leq \frac{m}{2} \\
2 \sum_{k=1}^{i-1} n_{k}+n_{i}+7, & \frac{m}{2}+1 \leq i \leq m-1 .\end{cases} \\
& \mathrm{f}^{*}\left(u_{m} u_{1}\right)=\sum_{k=1}^{m} n_{k}+3 .
\end{aligned}
$$

The labels of edges $v_{i j} v_{i(j+1)}$ for $1 \leq i \leq \frac{m}{2}-1,1 \leq j \leq n_{i}-1$ and $v_{i n_{i}} v_{i 1}$ for $1 \leq i \leq \frac{m}{2}-1$ are given as in pervious case. Otherwise, the labels of remaining edges $v_{i j} v_{i(j+1)}$ for $\frac{m}{2} \leq i \leq m, 1 \leq j \leq n_{i}-1$ and $v_{i n_{i}} v_{i 1}$ for $\frac{m}{2} \leq i \leq m$ are given as follows:

$$
\mathrm{f}^{*}\left(v_{\frac{m}{2} j} v_{\frac{m}{2}(j+1)}\right)= \begin{cases}2 \sum_{k=1}^{\frac{m}{2}-1} n_{k}+5, & \mathrm{j}=1 \\ 2 \sum_{k=1}^{\frac{m}{2}-1} n_{k}+13, & \mathrm{j}=2 \\ 2 \sum_{k=1}^{\frac{m}{2}-1} n_{k}+15, & \mathrm{j}=3 .\end{cases}
$$

For $\frac{m}{2}+1 \leq i \leq m-1$.

$\mathrm{f}^{*}\left(v_{\left(\frac{m}{2}+1\right) j} v_{\left(\frac{m}{2}+1\right)(j+1)}\right)=\left\{\begin{array}{cl}2 \sum_{k=1}^{\frac{m}{2}} n_{k}+2 j+7, & 1 \leq j \leq \frac{n_{\left(\frac{m}{2}+1\right)}}{2}-1 \\ 2 \sum_{k=1}^{\frac{m}{2}} n_{k}+2 j+9, & \frac{n_{\left(\frac{m}{2}+1\right)}}{2} \leq j \leq n_{\left(\frac{m}{2}+1\right)}-1 .\end{array}\right.$

$\mathrm{f}^{*}\left(v_{\left(\frac{m}{2}+1\right) n_{\left(\frac{m}{2}+1\right)}} v_{\left(\frac{m}{2}+1\right) 1}\right)=2 \sum_{k=1}^{\frac{m}{2}} n_{k}+n_{\frac{m}{2}+1}+7$.

For $\frac{m}{2}+2 \leq i \leq m-1$.

$\mathrm{f}^{*}\left(v_{i j} v_{i(j+1)}\right)=\left\{\begin{array}{cc}2 \sum_{k=1}^{i-1} n_{k}+2 j-7, & 1 \leq j \leq \frac{n_{i}}{2}-1 \\ 2 \sum_{k=1}^{i-1} n_{k}+2 j+9, & \frac{n_{i}}{2} \leq j \leq n_{i}-1 .\end{array}\right.$

$\mathrm{f}^{*}\left(v_{i n_{i}} v_{i 1}\right)=2 \sum_{k=1}^{i-1} n_{k}+n_{i}+7$.

$\mathrm{f}^{*}\left(v_{m j} v_{m(j+1)}\right)= \begin{cases}\sum_{k=1}^{m} n_{k}+1, & j=1 \\ 1, & j=2 \\ 3, & j=3 .\end{cases}$

$\mathrm{f}^{*}\left(v_{m 4} v_{m 1}\right)=\sum_{k=1}^{m} n_{k}+3$. 
Case (III): When $m=2 k$ and $k$ is odd, we define $f$ as follows:

$$
\mathrm{f}\left(\mathrm{u}_{i}\right)= \begin{cases}2 n_{m}-2, & i=1 \\ 2 n_{m}+2 \sum_{k=1}^{i-1} n_{k}-2, & 2 \leq i \leq \frac{m}{2} \text { and } i \text { is odd } \\ 2 n_{m}+2 \sum_{k=1}^{i-1} n_{k}-8, & 2 \leq i \leq \frac{m}{2} \text { and } i \text { is even } \\ 2 \sum_{k=1}^{\frac{m}{2}} n_{k}+2, & i=\frac{m}{2} \\ 2 n_{m}+2 \sum_{k=1}^{i-1} n_{k}+2, & \frac{m}{2}+1 \leq i \leq m \text { and } i \text { is odd } \\ 2 n_{m}+2 \sum_{k=1}^{i-1} n_{k}, & \frac{m}{2}+1 \leq i \leq m \text { and } i \text { is even. }\end{cases}
$$

The labels of vertices $v_{i j}$ for $1 \leq i \leq \frac{m}{2}-2,1 \leq j \leq n_{i}$ and $v_{m j}$ for $1 \leq j \leq n_{i}$ are given as in Case(I). Now the labels of remaining vertices $v_{i j}$ for $\frac{m}{2}-1 \leq i \leq m-1,1 \leq j \leq n_{i}$ are given as follows:

$$
\begin{aligned}
& \mathrm{f}\left(\mathrm{v}_{\left(\frac{m}{2}-1\right) j}\right)= \begin{cases}2 n_{m}+2 \sum_{k=1}^{\frac{m}{2}-2} n_{k}+2 j-10, & 1 \leq j \leq n_{\left(\frac{m}{2}-1\right)} \text { and } j \text { is odd } \\
2 n_{m}+2 \sum_{k=1}^{\frac{m}{2}-2} n_{k}+2 j-2, & 1 \leq j \leq \frac{n_{\left(\frac{m}{2}-1\right)}}{2} \text { and } j \text { is even } \\
2 n_{m}+2 \sum_{k=1}^{\frac{m}{2}-2} n_{k}+2 j+2, & \frac{n_{\left(\frac{m}{2}-1\right)}}{2}+2 \leq j \leq n_{\left(\frac{m}{2}-1\right)} \text { and } j \text { is even. }\end{cases} \\
& \mathrm{f}\left(\mathrm{v} \frac{m}{2} j\right)= \begin{cases}2 \sum_{k=1}^{\frac{m}{2}} n_{k}+2 j, & 1 \leq j \leq n_{\frac{m}{2}} \text { and } j \text { is odd } \\
2 \sum_{k=1}^{\frac{m}{2}} n_{k}-8, & j=2 \\
2 \sum_{k=1}^{\frac{m}{2}} n_{k}+2 j-8, & 4 \leq j \leq \frac{n \frac{m}{2}}{2} \text { and } j \text { is even } \\
2 \sum_{k=1}^{\frac{m}{2}} n_{k}+2 j, & \frac{n \frac{m}{2}}{2}+2 \leq j \leq n_{\frac{m}{2}} \text { and } j \text { is even. }\end{cases}
\end{aligned}
$$

For $\frac{m}{2}+1 \leq i \leq m-1, i$ is even.

$$
\mathrm{f}\left(\mathrm{v}_{i j}\right)= \begin{cases}2 n_{m}+2 \sum_{k=1}^{i-1} n_{k}+2 j-2, & 1 \leq j \leq n_{i} \text { and } j \text { is odd } \\ 2 n_{m}+2 \sum_{\substack{i-1 \\ i=1}}^{i-1} n_{k}+2 j-2, & 1 \leq j \leq \frac{n_{i}}{2} \text { and } j \text { is even } \\ 2 n_{m}+2 \sum_{k=1}^{i=1} n_{k}+2 j+2, & \frac{n_{i}}{2}+1 \leq j \leq n_{i} \text { and } j \text { is even. }\end{cases}
$$

For $\frac{m}{2}+1 \leq i \leq m-1, i$ is odd. 


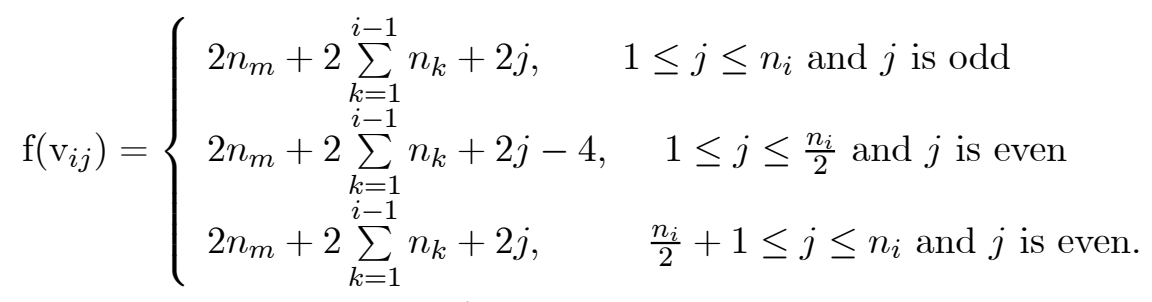

Hence, the edge labels of $A C l_{\left(m ; n_{1}, n_{2}, \ldots, n_{m}\right)}$ are given as follows:

$$
\mathrm{f}^{*}\left(u_{i} u_{(i+1)}\right)= \begin{cases}2 n_{m}+n_{1}-5, & i=1 \\ 2 n_{m}+2 \sum_{k=1}^{i-1} n_{k}+n_{i}-5, & 2 \leq i \leq \frac{m}{2}-2 \\ 2 n_{m}+2 \sum_{k=1}^{\frac{m}{2}-2} n_{k}+n_{\frac{m}{2}-1}-3, & i=\frac{m}{2}-1 \\ n_{m}+2 \sum_{k=1}^{\frac{m}{2}} n_{k}+1, & i=\frac{m}{2} \\ 2 n_{m}+2 \sum_{k=1}^{i-1} n_{k}+n_{i}+1, & \frac{m}{2}+1 \leq i \leq m-1 . \\ \mathrm{f}^{*}\left(u_{m} u_{1}\right)=2 n_{m}+\sum_{k=1}^{m-1} n_{k}-1 . & \end{cases}
$$

The labels of edges $v_{i j} v_{i(j+1)}$ for $1 \leq i \leq \frac{m}{2}-2,1 \leq j \leq n_{i}-1$, $v_{i n_{i}} v_{i 1}$ for $1 \leq i \leq \frac{m}{2}-2, v_{m j} v_{m(j+1)}$ for $1 \leq j \leq n_{i}-1$ and $v_{m n_{m}} v_{m 1}$ are given as in Case(I). Otherwise, the labels of remaining edges $v_{i j} v_{i(j+1)}$ for $\frac{m}{2}-1 \leq i \leq m-1,1 \leq j \leq n_{i}-1$ and $v_{i n_{i}} v_{i 1}$ for $\frac{m}{2}-1 \leq i \leq m-1$ are given as follows:

$$
\begin{aligned}
& \mathrm{f}^{*}\left(v_{\left(\frac{m}{2}-1\right) j} v_{\left(\frac{m}{2}-1\right)(j+1)}\right)= \begin{cases}2 n_{m}+2 \sum_{k=1}^{\frac{m}{2}-2} n_{k}+2 j-5, & 1 \leq j \leq \frac{n_{\left(\frac{m}{2}-1\right)}}{2} \\
2 n_{m}+2 \sum_{k=1}^{\frac{m}{2}-2} n_{k}+2 j-3, & \frac{n_{\left(\frac{m}{2}-1\right)}}{2}+1 \leq j \leq n_{\left(\frac{m}{2}-1\right)}-1 . \\
\mathrm{f}^{*}\left(v_{\left(\frac{m}{2}-1\right) n_{\left(\frac{m}{2}-1\right)}} v_{\left(\frac{m}{2}-1\right) 1}\right)=2 n_{m}+2 \sum_{k=1}^{\frac{m}{2}-2} n_{k}+n_{\frac{m}{2}-1}-3 .\end{cases} \\
& \mathrm{f}^{*}\left(v_{\frac{m}{2} j} v_{\frac{m}{2}(j-1)}\right)= \begin{cases}2 \sum_{k=1}^{\frac{m}{2}} n_{k}-3, & j=1 \\
2 \sum_{k=1}^{\frac{m}{2}} n_{k}-1, & j=2 \\
2 \sum_{k=1}^{\frac{m}{2}} n_{k}+2 j-3, & 3 \leq j \leq \frac{n_{\frac{m}{2}}}{2} \\
2 \sum_{k=1}^{\frac{m}{2}} n_{k}+2 j+1, & \frac{n \frac{m}{2}}{2}+1 \leq j \leq n_{\frac{m}{2}}-1 .\end{cases}
\end{aligned}
$$


$\mathrm{f}^{*}\left(v_{\frac{m}{2}} n_{\frac{m}{2}} v_{\frac{m}{2}} 1\right)=2 \sum_{k=1}^{\frac{m}{2}} n_{k}+n_{\frac{m}{2}}+1$.

For $\frac{m}{2}+1 \leq i \leq m-1$.

$\mathrm{f}^{*}\left(v_{i j} v_{i(j+1)}\right)= \begin{cases}2 n_{m}+2 \sum_{k=1}^{i-1} n_{k}+2 j-1, & 1 \leq j \leq \frac{n_{i}}{2} \\ 2 n_{m}+2 \sum_{k=1}^{i-1} n_{k}+2 j+1, & \frac{n_{i}}{2}+1 \leq j \leq n_{i}-1 .\end{cases}$

$\mathrm{f}^{*}\left(v_{i n_{i}} v_{i 1}\right)=2 n_{m}+2 \sum_{k=1}^{i-1} n_{k}+n_{i}+1$.

Illustration 3.2. In the following Figure 6, Figure 7 and Figure 8 we show an even vertex odd mean labeling on arbitrary calendula graphs $A C l_{(12 ; 8,12,8,12,16,4,8,16,8,12,12,4)}, A_{C l} l_{(8 ; 4,12,4,8,4,12,4,8)}$ and $A C l_{(10 ; 12,4,8,12,8,12,12,4,8,8)}$ respectively.

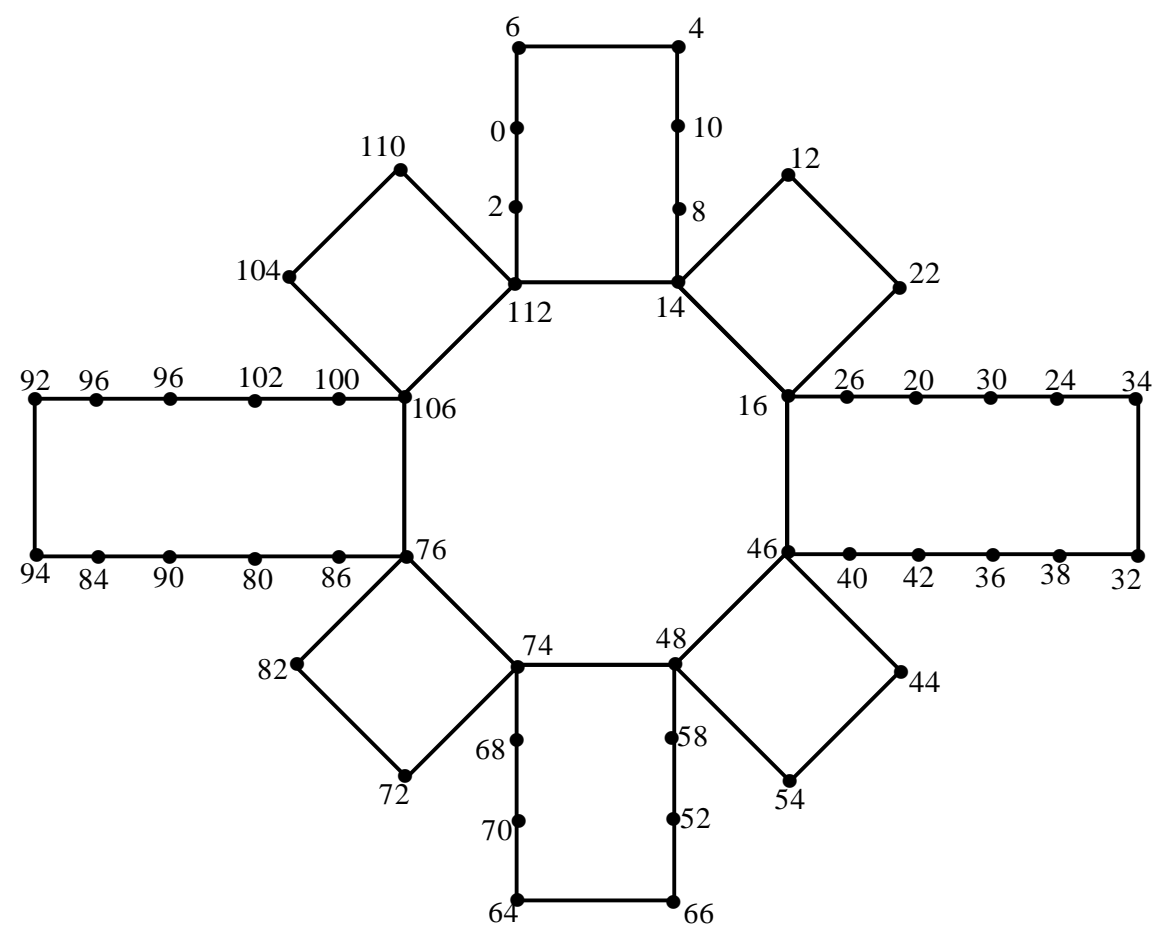

Figure 3.2: An even vertex odd mean graph of $A C l_{(8 ; 4,12,4,8,4,12,4,8)}$ 


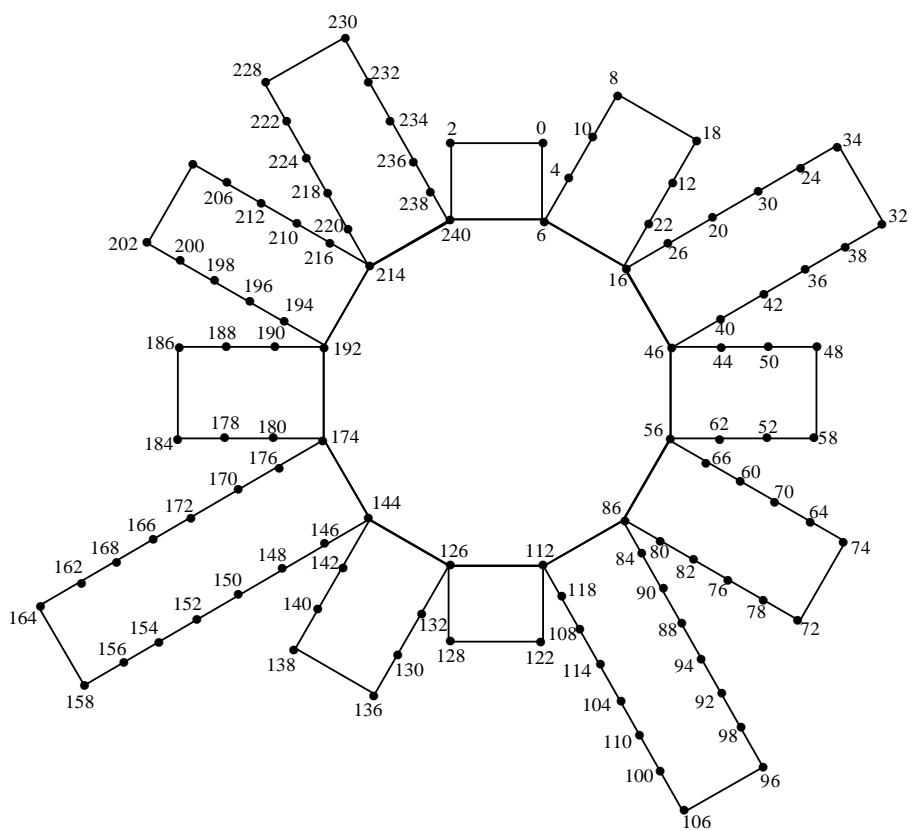

Figure 3.3: An even vertex odd mean graph of $A C l_{(12 ; 8,12,8,12,16,4,8,16,8,12,12,4)}$

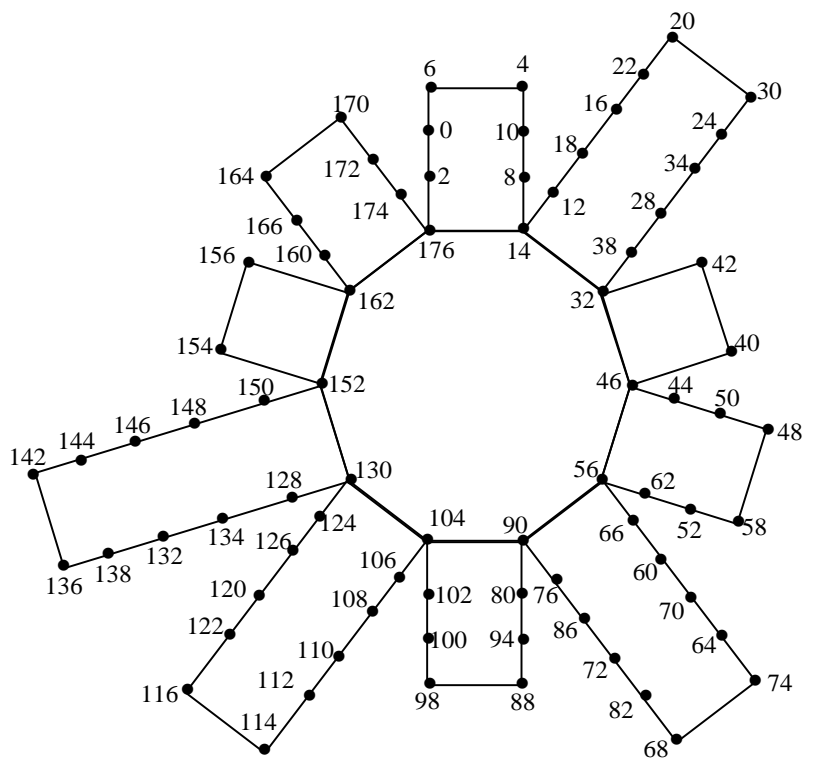

Figure 3.4: An even vertex odd mean graph of $A C l_{(10 ; 12,4,8,12,8,12,12,4,8,8)}$ 


\section{References}

[1] J. A. Gallian, "Graph labeling", The electronic journal of combinatorics, vol.17, no. DS6, Nov. 2010, doi: 10.37236/ 27

[2] F. Harary, Graph theory, Reading, MA: Addison-Wesley, 1972.

[3] M. Basher, "Further results on even vertex odd mean graphs", Journal of discrete mathematical sciences and cryptography, Nov. 2019, doi: 10.1080/ 09720529.2019.1675301

[4] P. Jeyanthi, D. Ramya, and M. Selvi, "Even vertex odd mean labeling of transformed trees", TWMS journal appied engineering mathematics, vol. 10, no. 2, pp. 338-345, 2020. [On line]. Available: https:/ / bit.ly/ 3kL2KiN

[5] A. Rosa, "On certain valuations of the vertices of a graph", in International Symposium on Graph Theory and its Applications, held in Rome, July 1966 under the auspices of the International Computation Center (Rome), P. Rosenstiehl, Ed. London: Gordon \& Breach, 1967, pp. 349-355. [On line]. Available: https:/ / bit.ly/ 320qqst

[6] T. R. Pradipta and A. N. M. Salman, "Some cycle-supermagic labelings of the calendula graphs", Journal of physics: conference series, vol. 948, Art ID. 012071, 2018, doi: 10.1088/ 1742-6596/948/ 1/ 012071

[7] R. Vasuki, A. Nagarajan and S. Arockiaraj, "Even vertex odd mean labeling of graphs", SUT journal of mathematics, vol. 49, no. 2, pp. 79-92, 2013. 